\title{
Implementing self-administration of insulin in hospital: a journey of discovery and innovation. Part 1: Culture and storage
}

\author{
VICKI L ROWSE
}

\begin{abstract}
Patients with diabetes routinely manage their insulin at home, but when they are admitted to hospital it is common practice to take their insulin away and store it in the ward fridge. Medicines rounds and mealtimes are poorly aligned and, as a result, patients can have delayed doses and increased hypo- and hyperglycaemic episodes. Best practice states that patients should be offered self-administration of insulin, but it is not routine in most trusts. This paper reports on a project to increase the number of patients assessed and supported to administer their insulin in hospital, the reasons why the project was initially unsuccessful and the challenges of changing culture and beliefs around insulin administration. A second paper discusses steps taken to support changes in hospital trusts.

Br J Diabetes 2018;18:18-21
\end{abstract}

Key words: insulin, inpatients, safety, culture

\section{Introduction}

Self-care and empowerment are two aims of modern healthcare for patients with long-term conditions. Patients with diabetes who are dependent on insulin are educated and supported to manage their condition in their everyday lives. But when they are admitted to hospital we routinely take their insulin away from them, effectively removing their ability to self-care because of safety concerns about the misuse of insulin. Ironically, this has led to patients often having poorer control in hospital than at home. They also experience an increase in hypo- and hyperglycaemic episodes, ${ }^{1}$ and errors including delayed and missed doses, wrong insulin and wrong dose.

There has been significant work undertaken nationally to improve insulin safety, but one potential solution - supporting patients to administer their own insulin while in hospital - has had little

Wessex Academic Health Science Network, The Innovation Centre, Chilworth, Hampshire, UK

Address for correspondence: Ms Vicki L Rowse Senior Programme Manager Medicines Optimisation and Atrial Fibrillation Programmes, Wessex Academic Health Science Network, The Innovation Centre, 2 Venture Road, Chilworth, Hampshire SO16 7NP, UK

Tel: +44 (0)2382020840

E-mail:vicki.rowse@wessexahsn.net

https://doi.org/10.15277/bjd.2018.160 success in getting embedded as everyday practice. Wessex Academic Health Science Network (AHSN) was asked to lead a local project to increase the number of people offered and supported to administer their own insulin in hospital. In this two-part article we describe our journey, what we discovered and how we innovated to support change.

\section{Background}

There are 3 million people who have been diagnosed with diabetes in England (6.2\% of the population). ${ }^{2}$ People with diabetes are twice as likely to be admitted to hospital, to have longer stays $(80,000 \text { excess bed days per year })^{3}$ and be admitted overnight for procedures that can be done as day cases.

One in six hospital beds is occupied by someone who has diabetes, and in some hospitals it is as many as $40 \% .^{2}$ One in four people admitted to hospital with heart failure, heart attack or stroke has diabetes. Diabetes Health Intelligence ${ }^{4}$ has shown a $6.4 \%$ increase in deaths for patients with diabetes in hospital compared with those without diabetes.

In recent years, the convictions of nurses Victorino Chua and Beverley Allitt for murder by deliberate misuse of insulin have resulted in the tightening of control of insulin in ward environments, thus making it much more difficult for patients to self-administer. Non-intentional incidents can also cause significant harm.

National drivers

The NICE quality standards for diabetes recommends that people with diabetes admitted to hospital are cared for by appropriately trained staff, provided with access to a specialist diabetes team, and given the choice of self-monitoring and managing their own insulin. 5

NHS Diabetes published a report entitled "Self-Management of Diabetes in Hospital" in March 2012 which stated:6

The aim of the document is to improve the safety of the in hospital management of diabetes. Diabetes care is very individualised, especially if that person is using insulin. It follows that the person with the greatest expertise in managing diabetes is commonly the individual themselves. Allowing patients to self-manage their diabetes in hospital should significantly improve patient safety. The correct support structures need to be provided to ensure that this is properly monitored, but this must be done without creating unnecessary bureaucracy. 
It is interesting that, five years later, there is still no uniform or standard approach.

It is important at this point to define self-management and selfadministration. Self- management describes a patient who monitors their blood glucose, decides on the insulin dose and administers the dose to themselves. Self-administration describes a patient who administers their insulin, but may not be undertaking self-management at that point in time. Our work has focused on self-administration of insulin.

\section{Wessex AHSN approach}

In 2014, seven local trusts were visited to determine current practice of supporting patients to administer their own insulin, and it was clear that practice differed within trusts and between trusts. A number of initiatives had been introduced to improve insulin safety including prescribing in units; banning abbreviations; mandating use of insulin-specific equipment to prevent wrong dose; and use of safety needles on pens. Ironically, some of these have led to other or increased risks, such as the drawing of insulin from cartridges which has resulted in a Patient Safety Alert. ${ }^{7}$ No trust had an embedded process for all patients using insulin to be offered and assessed for self-administration.

In January 2015, diabetes nurse specialists in two trusts started leading projects in their trusts, supported by the diabetes teams. However, by August, progress in both trusts was slow due to clinical pressures and difficulties in getting wider engagement across the trusts. They continued until the end of 2015 and then a review was undertaken which showed that there had been virtually no progress. In 2016, Wessex AHSN started development of an implementation guide, ${ }^{8}$ described in Part 2 , and funded project manager time to lead the work. During visits and discussions with staff in the seven trusts and the lack of progress in the two trusts who had specific projects to implement self-administration of insulin, two clear themes emerged: storage and culture.

\section{Storage}

Although the principle of self-medication in hospital is endorsed by regulatory bodies including the Nursing and Midwifery Council (NMC), registered general nurse (RGN), General Medical Council (GMC) and the Care Quality Commission (CQC), its practice is variable. In 2005, the Duthie Report entitled "The Safe and Secure Handling of Medicines" recommended:9

Safe and secure processes will be needed to ensure that the patient has controlled access to an adequate supply of the correct medicines, appropriately stored so that they are fit for use, and that the medicines cannot be subject to unauthorised removal eg, by other patients.

and

When schemes for self-administration of medicines and/or 'one-stop dispensing' are in operation on the ward, each patient involved in the scheme should have a lockable receptacle for medicines (eg, drawer, individual cupboard) which is not readily portable.
This has been interpreted in different ways by organisations, with some giving the patient responsibility for storing their medicine out of sight and others not allowing any form of self-administration as they do not have locked storage at the bedside that they will let the patient access.

Concerns raised by trusts include:

- Patients lose the Patient's Own Drugs cupboard (POD) keys

- The POD contains other medicines that we do not want the patient to administer

- CQC does not like key systems as keys can be taken by other people and then used

- Getting appropriate storage is too expensive

- Lockable boxes are portable

The regulations plus the cost of purchasing lockers suitable for storage of patients' self-administered medicines were cited in all trusts as a barrier to trust-wide implementation of self-administration.

\section{Culture}

Traditionally, nurses are responsible for the administration of medicines. The idea of patient self-administration raises fears about accountability, and concerns that nurses could lose their registration if the patient makes a mistake or someone else misuses the medicine. Concerns have also been expressed about suitability of patients for self-administration, and that it is difficult to keep track as their suitability may change during their admission.

The $\mathrm{NMC}^{10}$ is clear that patients should be supported to selfadminister where appropriate, and gives guidance on what the nurse should do. Key statements are:

- Standard 5: The NMC welcomes and supports the self-administration of medicinal products and the administration of medication by carers wherever it is appropriate.

- Standard 6: To assess patients on a regular basis using local policies to ensure that the individual patient is still able to selfadminister.

The standards also give three levels of suitability for patient selfadministration (Table 1), and these were found to be commonly used in Wessex.

In addition, the NMC is clear that the nurse has responsibility for

Table 1 Patient assessment for self-administration suitability at three levels: NMC Standards for Medicines Management 2007 (updated 2010) ${ }^{10}$

Level 1 The registrant is responsible for the safe storage of the medicinal products and the supervision of the administration process, ensuring the patient understands the medicinal product being administered

Level 2 The registrant is responsible for the safe storage of the medicinal products. At administration time, the patient will ask the registrant to open the cabinet or locker. The patient will then self-administer the medication under the supervision of the registrant

Level 3 The patient accepts full responsibility for the storage and administration of the medicinal products. The registrant checks the patient's suitability and compliance verbally

The level should be documented in the patient's notes 
initial and continued assessment of patients administering their own medicines, and acting on changes in the patient's condition:

Whilst the registrant has a duty of care towards all patients, the registrant is not liable if a patient makes a mistake selfadministering as long as the assessment was completed as the local policy describes and appropriate actions were taken to prevent re-occurrence of the incident.

Audits in three trusts showed that $50 \%$ of patients (17/34 on 36 wards) who were taking insulin with meals were self-administering. None of these patients had documented risk assessments. None of these patients had documented risk assessments and all of these patients had demanded self-administration. Where diabetes teams had implemented self-administration of insulin, they found that assessment documentation was not consistently used due to staff turnover and busy wards.

\section{Trust processes}

Whilst the majority of trusts have self-administration policies in place, risk assessments and documentation to support implementation of the policies are not uniformly applied. As a result, there is confusion and concern about patient self-administration of medicines.

Trust processes around meals and medicine rounds also cause problems for insulin-dependent patients. The number of inpatient severe hypoglycaemic episodes (1/50) and diabetic ketoacidosis/ hyperglycaemic hyperosmolar state (DKA/HHS) episodes (1/200) have remained unaltered over the three years of National Diabetes Inpatient Audits (NaDIA). ${ }^{1}$ This is often due to medicines rounds and mealtimes not being coordinated.

\section{Best practice}

Key to getting this right as an organisation is being able to demonstrate that the risks have been identified and systems put in place to mitigate those risks, whilst promoting independence and better care. The CQC supports patients administering their own medicines. and have observed that where it is not being done well the common themes are:

- No formal risk assessment around how insulin is to be selfadministered

- No arrangements in place for the safe storage of insulin

- No reference on the chart to the fact that the person is administering his or her own insulin

- Lack of clinical supervision of the self-administration

- No recording or incorrect recording of the administration on the drug chart ${ }^{11}$

Local projects were commenced in one teaching hospital and one district general hospital. These were led by the diabetes teams who were very enthusiastic that this was the right thing to do. We spoke to patients on wards and at a Diabetes UK meeting to get their views and they told us they felt disempowered by giving up their insulin. No one described a positive experience. A patient who had insisted on self-managing his insulin told us his story (Figure 1), which gave insight into the challenges.
Figure 1. A patient story.
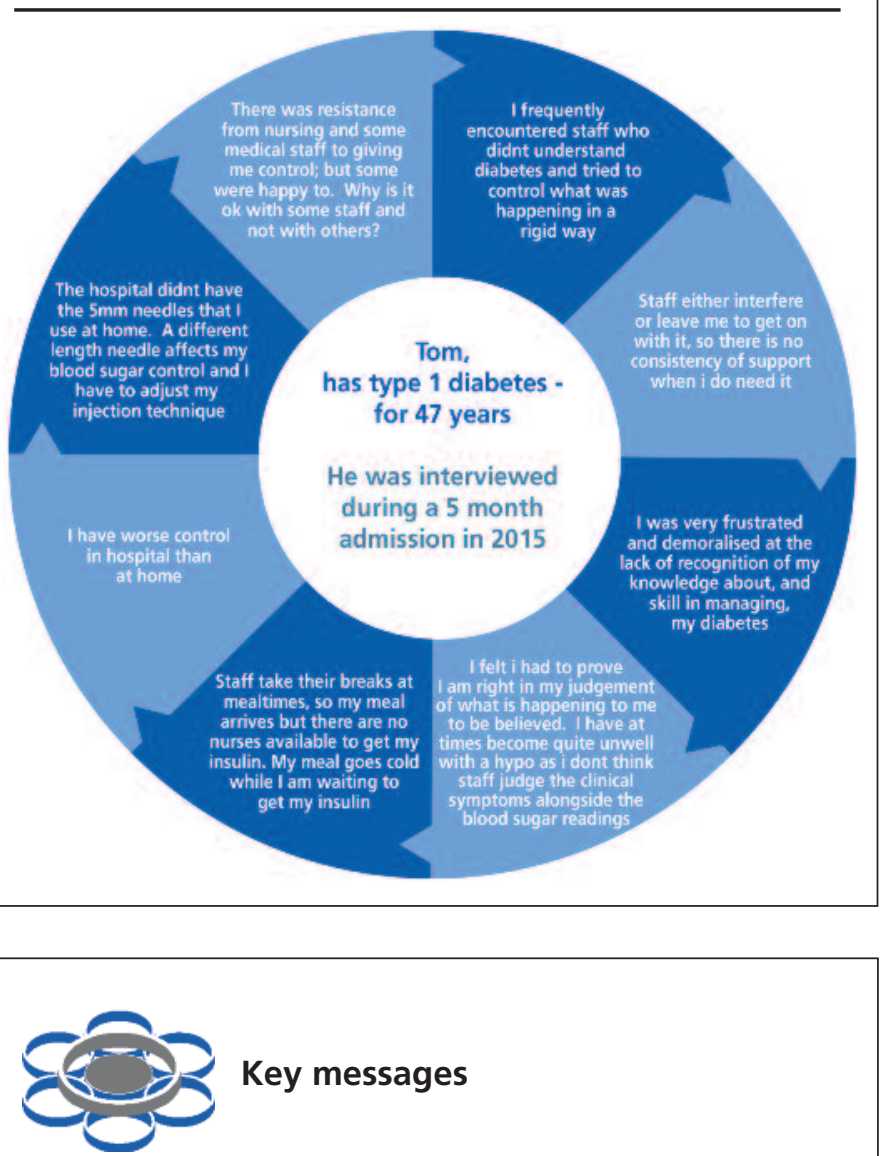

- Patients want to have the choice to administer their own insulin and to be supported to do so

- Many patients are already self-administering insulin without proper risk assessments or support

- Nurses do not always understand their responsibilities in respect of self-administration

- Storage and ward culture are two key barriers

- Current practices for administering insulin cause harm through error and delayed doses

However, in both trusts progress was limited, primarily because of culture and storage issues outlined earlier. It became apparent that the diabetes team could not undertake this work on top of their 'day job' and wider trust involvement was required. It also became apparent that there was a lack of knowledge of how to undertake a major change in management, and it was decided to develop an implementation guide and to fund dedicated project manager time to move the projects forward. This will be described in the second article.

\section{Summary}

Current practices for administering insulin are causing harm to patients through error and delayed doses. Guidance and best practice promote self-care, and support for patients to self-administer 
insulin while in hospital is in patients' best interests. However, they must be properly assessed and supported to do so. Our second article describes the Guide that was developed to support the change process, reports our experience of implementation and provides some guidance to support other trusts to introduce selfadministration of insulin. ${ }^{8}$

\section{Timeline}

2014 April to December: visits to seven trusts to scope practice and experience of self-administration

2015 January: commenced working with two trusts. Diabetes nurse specialists leading projects

2015 August: progress slow due to clinical pressures and difficulties in getting wider engagement, data collected by individual trusts on insulin errors

2016 January: review of both projects. Very little progress. Decision made to develop Guide to Implementation and to source project manager funding for dedicated time.

\section{Conflict of interest: None Funding: None}

\section{References}

1. Diabetes UK. Reducing diabetes harms in hospital: National Diabetes Mortality and Morbidity Project. Key themes from a learning event with Diabetes Mortality and Mortality project participants, 13 February 2015. http://www.disn-uk.co.uk/course-feedback/untitled

2. Diabetes UK. The State of the Nation. 2016. . https://diabetes-resourcesproduction.s3-eu-west-1.amazonaws.com/diabetes-storage/migration/ pdf/Diabetes\%2520UK\%2520State\%2520of\%2520the\%2520Nation\%25202016.pdf

3. Sampson MJ, Crowle T, Dhatariya K, et al. Trends in bed occupancy for inpatients with diabetes before and after the introduction of a diabetes inpatient specialist nurse service. Diabet Med 2006;23:1008-115. http://dx.doi.org/10.1111/j.1464-5491.2006.01928.x

4. Yorkshire and Humber Public Health Observatory Diabetes Health Intelligence. Mortality among inpatients with diabetes: key findings for England. May 2012. http://webarchive.nationalarchives.gov.uk/ 20170302112650/http://www.yhpho.org.uk/resource/view.aspx?RID=1 39172 (accessed 16 June 2017).

5. National Institute for Health and Care Excellence. Diabetes in adults. Quality Standard [QS6]. 2011 (updated 2016). https://www.nice.org.uk/ guidance/qs6 (accessed 16 June 2107).

6. NHS Diabetes. Joint British Diabetes Societies for Inpatient Care Group. Self-management of diabetes in hospital. March 2012. https://www. diabetes.org.uk/Professionals/Position-statements-reports/Specialist-carefor-children-and-adults-and-complications/Self-management-of-diabetes-in-hospital/ (accessed 16 June 2017)

7. NHS Improvement. Patient Safety Alert. Risk of severe harm and death due to withdrawing insulin from pen devices. 16 November 2016 NHS/PSA/W/2016/011. https://improvement.nhs.uk/news-alerts/risk-severe-harm-and-death-withdrawing-insulin-pen-devices/ (accessed 16 June 2017).

8. Wessex Academic Health Science Network. Self-administration of insulin in hospital: a guide to support Trusts through the implementation process. 2017. http://wessexahsn.org.uk/projects/58/self-administrationof-insulin-in-hospital (accessed 16 June 2017).

9. Royal Pharmaceutical Society. The safe and secure handling of medicines: a team approach. A revision of the Duthie Report (1988) led by the Hospital Pharmacists' Group of the Royal Pharmaceutical Society. March 2005. https://www.rpharms.com/Portals/O/RPS\%20document\%20library/Open \%20access/Publications/Safe $\% 20$ and $\% 20$ Secure $\% 20 \mathrm{Han}$ dling\%20of\%20Medicines\%202005.pdf (accessed 16 June 2017).

10. Nursing and Midwifery Council. Standards for Medicines Management 2007 (updated 2010). http://www.nmc.org.uk/standards/additionalstandards/standards-for-medicines-management/ (accessed 16 June 2017).

11. Billington S. Head of Medicines Optimisation, Primary Medical Services and Integrated Care Directorate, Care Quality Commission. By email 16 August 2017 and 16 July 2015. 